\title{
Laparoscopy-assisted resection of proximal gastric cancer: is less than all more or less complete, or is all more, nonetheless?
}

\author{
Roderich E. Schwarz $\cdot$ Changhua Zhang • \\ John C. Mansour
}

Published online: 18 December 2012

(c) The International Gastric Cancer Association and The Japanese Gastric Cancer Association 2012

In this issue of Gastric Cancer, Ahn et al. [1] present a series of 131 patients with early-stage proximal gastric cancer who underwent laparoscopic resection, either in the form of laparoscopy-assisted total gastrectomy (LATG) or that of laparoscopy-assisted proximal gastrectomy (LAPG). The comparison of these two minimally invasive techniques with assessment of multiple early and late measures of outcomes is intriguing, and although not being based on a randomized trial deserves careful recognition and analysis. Fifty LAPG patients had lower intraoperative blood loss and operative times than 81 patients after LATG, but no obvious differences of early postoperative recovery or longer-term survival outcomes were found, with the main group differences consisting of delayed complications and functional parameters. The results generally appear to be excellent, with good postoperative outcomes indicative of proper patient selection and appropriate conduct of the operative procedures. With respect to a comparison of proximal versus total gastrectomy in a laparoscopy-assisted approach, these findings seem to resemble those results found after an open approach to total and proximal gastrectomy that showed no differences between the techniques regarding early postoperative and late oncological outcomes, but indicated possible functional differences between these groups [2]. Are the authors just wrapping the

This editorial refers to the article doi:10.1007/s10120-012-0178-x.

R. E. Schwarz · C. Zhang · J. C. Mansour

Division of Surgical Oncology, UT Southwestern Cancer Center, Dallas, TX 75390, USA

R. E. Schwarz $(\bowtie)$

Department of Surgery, UT Southwestern Medical Center,

5323 Harry Hines Blvd., Dallas, TX 75390-8548, USA

e-mail: Roderich.Schwarz@utsouthwestern.edu old debate on total versus proximal gastrectomy for proximal gastric cancer into a minimally invasive package here, or is their experience telling us more? We think that this series highlights several relevant aspects and poses some questions that deserve further comment.

First, Ahn and colleagues have of course accomplished this minimally invasive approach with an admirable safety and morbidity record, even for Asian standards, which tells us that LATG and LAPG appear to be acceptable procedures for early-stage gastric cancer regarding safety and oncological adequacy. This is not too surprising now, as we have learned from other comparative efforts that minimally invasive approaches to distal gastric cancer can result in similar postoperative morbidity [3], recurrence patterns [4], and overall survival that is at least not inferior to traditional open resections [5]. If performed in the setting of proper expertise, a laparoscopy-assisted technique appears to be an acceptable choice for proximal gastric cancer treatment from this standpoint.

Second, an important question appears to be how the LAPG or LATG techniques were chosen. Were there differences in patient presentation that led to the choice of one technique over the other, or were there surgeon-based preferences or biases toward one or the other approach? Because the study is not based on a randomized trial or case-matched comparisons, have the authors developed expertise during this study period in which setting one technique is superior to the other? If we assume that the patient cohorts undergoing LAPG and LATG are not dissimilar, how do the authors explain that the proximal margin length is different between the groups? If they still perform LAPG in this setting, how have they addressed the implied need for anti-reflux measures, and if so, what are the results? Provided one can address reflux-related issues after LAPG with a procedure modification, should 
the reader choose LAPG over LATG under certain circumstances?

Third, can we be satisfied that LAPG and LATG lead to oncologically equivalent results? Oncological equivalence of proximal gastrectomy compared to total gastrectomy has been advocated by some [2] but challenged by others [6]. Ahn et al. do not provide us with data that suggest equivalent outcomes in this respect, and this question can therefore not be answered. Although the overall survival appears similar, disease-specific events should be analyzed and reported to shed proper light on this issue. We recognize that the likelihood for recurrence in the early gastric cancer setting of this study is relatively low, but we also must be aware of the smaller tumor size in the LAPG group, which could translate into a lower hazard for recurrence or death. In addition, as mentioned, the proximal margin distance was smaller in the LAPG group, although one should not assume that this is truly necessitated by the different technique. Does the distal margin, by definition smaller after LAPG, lead to more frequent local recurrences? Should the lower total lymph node count after LAPG be a concern? Some studies indicate a correlation of total nodal counts and survival outcomes for early-stage and for late-stage gastric cancer [7, 8]. In general, we interpret the total lymph node counts obtained in the series of Ahn and colleagues after LAPG and after LATG to be quite sufficient. Sentinel lymph node navigation has been described in the LAPG setting and may become a useful tool for determining the regional dissection needs in the future [9].

Fourth, functional outcomes, especially in the longer term, appear to be different after LAPG and LATG in the results presented. There is clearly a greater risk of reflux after a distal stomach-preserving resection as observed after LAPG, and the predominantly bile reflux-related challenges have been described by others $[6,10]$. Severity and frequency of reflux appears to be related to the reconstruction rather than the type of resection [11]. Although the authors have nicely attempted to dissect their learning experience in general and their insight with sideto-side esophagogastrectomy after LAPG within this manuscript, which appears to be a result of a balance between development of either stenoses or reflux problems in the primary anastomoses applied, it remains unanswered whether other reconstruction techniques may not be more effective in preventing reflux after LAPG. One example would be a small bowel interposition between esophagus and gastric remnant as popularized by Merendino and others [12], although its ability to control biliary reflux is not uniformly confirmed [13]. Of note, a Merendino interposition in conjunction with vagal nerve preservation can be performed laparoscopically [14] and perhaps should be considered after LAPG. Other published experiences with reconstruction after LAPG include a side-stapler overlap technique [15], a Toupet partial gastric wrap [16], or the attempt to preserve the lower esophageal sphincter [17], but the generally favorable outcomes reported are based on smaller case series and can therefore not yet provide a definitive solution to this question. Furthermore, should we be surprised that LAPG did not lead to more obvious nutritional or other functional advantages than LATG? This accomplishment would perhaps require more specific endpoints to be analyzed than those provided by Ahn et al., including weight stability over longer time period, frequency of diarrhea and bowel movements, onset of osteoporosis, anemia, or mineral or vitamin deficiencies [18]. With the data provided, obvious advantages of LAPG regarding these domains cannot be supported.

Finally, what are the potential implications with respect to these two techniques for the application for adjuvant chemotherapy, especially when given preoperatively? Although not accepted as standard of care in all parts of the world, preoperative chemotherapy or postoperative chemoradiation for $\mathrm{T} 2$ and later-stage gastric cancers is rather widely applied in Western centers [19, 20]. Although Ahn et al. present a series in which these components had not been utilized, it does appear sensible to assume that there will be no significant differences between the techniques when it comes to early recovery after preoperative induction therapy, or regarding the ability to deliver postoperative adjuvant therapy, as this seems to depend mostly on the course of early postoperative recovery. If minimally invasive gastrectomy approaches would be able to lead to lower severe postoperative morbidity than open approaches, planned postoperative adjuvant therapy options may benefit from this approach.

What then are the conclusions that can be drawn from this manuscript? When it comes to laparoscopy-assisted resection of proximal gastric cancer, is less than all more or less complete, or is all more, nonetheless? We think that the answer is that less is not necessarily more, all appears slightly more, but all is not complete, either, and a final verdict is perhaps still pending. Ahn and colleagues should be congratulated for contributing to the development, the comparative analysis, and the discussion of these techniques. Based on their data, proponents of proximal gastrectomy or LAPG should be prepared to address refluxrelated challenges. Proponents of total gastrectomy in this setting will likely find little evidence to change their mind. As the quest for the optimal locoregional therapy of earlystage proximal gastric cancer continues, authors and readers alike who carry an interest in proximal and/or total gastrectomy should be encouraged to analyze these procedures in a randomized controlled prospective trial setting with inclusion of long-term metrics of functional, nutritional, and quality-of-life outcomes. 


\section{References}

1. Ahn SH, Lee JH, Park DJ, Kim HH. Comparative study of clinical outcomes between laparoscopy-assisted proximal gastrectomy (LAPG) and laparoscopy-assisted total gastrectomy (LATG) for proximal gastric cancer. Gastric Cancer 2012. doi: 10.1007/s10120-012-0178-x

2. Harrison LE, Karpeh MS, Brennan MF. Total gastrectomy is not necessary for proximal gastric cancer. Surgery (St. Louis). 1998;123(2):127-30.

3. Kim HH, Hyung WJ, Cho GS, et al. Morbidity and mortality of laparoscopic gastrectomy versus open gastrectomy for gastric cancer: an interim report-a phase III multicenter, prospective, randomized trial (KLASS Trial). Ann Surg. 2010;251(3):417-20.

4. Song J, Lee HJ, Cho GS, et al. Recurrence following laparoscopyassisted gastrectomy for gastric cancer: a multicenter retrospective analysis of 1,417 patients. Ann Surg Oncol. 2010;17(7):1777-86.

5. Park do J, Han SU, Hyung WJ, et al. Long-term outcomes after laparoscopy-assisted gastrectomy for advanced gastric cancer: a large-scale multicenter retrospective study. Surg Endosc. 2012; 26(6):1548-53.

6. Yoo $\mathrm{CH}$, Sohn BH, Han WK, Pae WK. Long-term results of proximal and total gastrectomy for adenocarcinoma of the upper third of the stomach. Cancer Res Treat. 2004;36(1):50-5.

7. Smith DD, Schwarz RR, Schwarz RE. Impact of total lymph node count on staging and survival after gastrectomy for gastric cancer: data from a large US-population database. J Clin Oncol. 2005;23(28):7114-24.

8. Schwarz RE, Smith DD. Clinical impact of lymphadenectomy extent in resectable gastric cancer of advanced stage. Ann Surg Oncol. 2007;14(2):317-28.

9. Takeuchi H, Oyama T, Kamiya S, et al. Laparoscopy-assisted proximal gastrectomy with sentinel node mapping for early gastric cancer. World J Surg. 2011;35(11):2463-71.

10. Katsoulis IE, Robotis JF, Kouraklis G, Yannopoulos PA. What is the difference between proximal and total gastrectomy regarding postoperative bile reflux into the oesophagus? Dig Surg. 2006; 23(5-6):325-30.

11. Shiraishi N, Adachi Y, Kitano S, Kakisako K, Inomata M, Yasuda K. Clinical outcome of proximal versus total gastrectomy for proximal gastric cancer. World J Surg. 2002;26(9):1150-4.

12. Moylan JP Jr, Bell JW, Cantrell JR, Merendino KA. The jejunal interposition operation: a follow-up on seventeen patients followed 10 to 17 years. Ann Surg. 1970;172(2):205-11.

13. Linke GR, Borovicka J, Tutuian R, et al. Altered esophageal motility and gastroesophageal barrier in patients with jejunal interposition after distal esophageal resection for early stage adenocarcinoma. J Gastrointest Surg. 2007;11(10):1262-7.

14. Pring C, Dexter S. A laparoscopic vagus-preserving Merendino procedure for early esophageal adenocarcinoma. Surg Endosc. 2010;24(5):1195-9.

15. Tsujimoto H, Uyama I, Yaguchi Y, et al. Outcome of overlap anastomosis using a linear stapler after laparoscopic total and proximal gastrectomy. Langenbecks Arch Surg. 2012;397(5):833-40.

16. Sakuramoto S, Yamashita K, Kikuchi S, et al. Clinical experience of laparoscopy-assisted proximal gastrectomy with Toupet-like partial fundoplication in early gastric cancer for preventing reflux esophagitis. J Am Coll Surg. 2009;209(3):344-51.

17. Kim DJ, Lee JH, Kim W. Lower esophageal sphincter-preserving laparoscopy-assisted proximal gastrectomy in patients with early gastric cancer: a method for the prevention of reflux esophagitis. Gastric Cancer 2012.

18. Baker A, Wooten LA, Malloy M. Nutritional considerations after gastrectomy and esophagectomy for malignancy. Curr Treat Options Oncol. 2011;12(1):85-95.

19. Cunningham D, Allum WH, Stenning SP, et al. Perioperative chemotherapy versus surgery alone for resectable gastroesophageal cancer. N Engl J Med. 2006;355(1):11-20.

20. Smalley SR, Benedetti JK, Haller DG, et al. Updated analysis of SWOG-directed intergroup study 0116: a phase III trial of adjuvant radiochemotherapy versus observation after curative gastric cancer resection. J Clin Oncol. 2012;30(19):2327-33. 